\title{
LA VERDAD \\ COMUNICATIVA \\ COMO RETO \\ CREATIVO EN KARL \\ JASPERS
}

Paula Arizpe*

La comunicación es el modo como se revela la verdad en el tiempo. ${ }^{1}$

Para Jaspers, la verdad es comunicación, entendida no como la mera transmisión de contenidos sino como la realización de la propia existencia y la realización existencial de los otros. Jaspers entiende la comunicación como un evento creativo dentro del cual se conoce la realidad a través de la propia realización.

El acceso a la verdad personal se conquista mediante las diversas perspectivas bajo las cuales ésta se aprehende. El punto de partida de esta aventura tanto existencial como cognoscitiva es la dicotomía kantiana entre sujeto y objeto. Este hecho escinde el conocimiento en perspectivas diversas, con lo que, en un primer momento, el significado de la verdad dependerá de la perspectiva bajo la cual el sujeto aprehenda los objetos del mundo. Al final, los distintos significados de la verdad confluyen en una unidad por encima del tiempo que el autor denomina trascendencia. La articulación racional de estos significados en un espacio y tiempo determinados la realiza la Existenz-sujeto

* Departamento Académico de Estudios Generales, ITAM.

${ }^{1}$ Jaspers, K., La fe filosófica, 1968, Buenos Aires, Losada, p. 43. 
PAULA ARIZPE

pleno en comunicación con el mundo- lo que le posibilita sostener un compromiso existencial con la verdad como totalidad. Para Jaspers la realización del ser sí mismo significa un compromiso radical con la verdad, lo cual otorga sentido a la existencia guiándola hacia la trascendencia. Éste es el reto al que Jaspers invita: "la creencia del hombre en su propia posibilidad".

\section{Una metodología de la apertura}

El autor establece que el saber humano va más allá del conocimiento científico, sus exigencias gnoseológicas pasan por el conocimiento científico del mundo pero van más allá puesto que pretende llegar al verdadero ámbito humano en el cual puede darse la comunicación auténtica, aquel ámbito en el cual el hombre puede realizar su propia posibilidad, al tiempo que completa el mundo humano con su actividad creativa. Se trata de un ámbito más allá de la objetividad, es decir transobjetivo: el situs en el cual el hombre puede realizar su propia posibilidad, y es la realización propia o materialización de estas posibilidades personales las que estructuran a los individuos en una comunidad orgánica.

Analicemos los pasos paulatinos que distienden al mundo hasta la profundidad genuinamente humana que el autor exige para el desarrollo de la libertad humana.

- El sujeto, en tanto conciencia empírica se encuentra ante un mundo de objetos mediante los cuales puede satisfacer sus necesidades básicas. Esta relación permanece a un nivel puramente sensible, por lo que la verdad tiene un significado de mera utilidad y se mueve dentro de las categorías satisfactorio-insatisfactorio. Evidentemente ésa es una perspectiva demasiado pobre para el hombre como totalidad, de manera que si permanece anclado a esta ínfima verdad, experimenta una profunda

${ }^{2}$ Idem, p. 5. 
insatisfacción. Por lo que debe seguir adelante, profundizar su relación con el mundo.

- El sujeto como conciencia general es capaz de conceptualizar formalmente los objetos del mundo agrupándolos de manera tal que puede obtener resultados prácticos. Esta formalización constituye una relación con el mundo desde una perspectiva más inmaterial, por lo que la verdad toma así un significado apodíctico dentro de las categorias consistente-inconsistente, dentro-fuera del sistema. Llegados a este punto la comunicación es lo suficientemente precisa como para agrupar a los miembros de la sociedad en torno a logros materiales, dentro de los cuales los individuos pueden ser sustituidos indistintamente, por lo cual, la comunicación a este nivel no es del todo humanamente satisfactoria. De este modo, para acceder a la comunicación existencial el hombre debe ir más allá en la relación comunicativa. ${ }^{3}$

- El sujeto en tanto espíritu es capaz de escoger ideales. En esta etapa enfrenta la decisión y descubre el reino de la libertad, que puede dirigir hacia las metas que se proponga después de definir su propia orientación intramundana. Este ámbito se acerca más al ámbito humano, por lo que la verdad aquí toma el significado de claridad dentro de las categorías intrínsecoextrínseco. Sin embargo, el hombre como totalidad aún debe dirigir su decisión hacia la trascendencia.

- Con este método Jaspers pretende guiar al hombre en tanto existencia posible hasta las profundidades de la comunicación existencial, en donde la verdad toma la forma de creencia en

3 “La filosofia sostiene la pretensión de captar el sentido de la vida más allá de todos los fines de este mundo, de manifestar el sentido que estos fines engloban; cumplir realizándolo en el presente este sentido, como si dijéramos transversalmente en la vida - con su propia presencia servir al propio tiempo y al futuro - no rebajar jamás al hombre o a un hombre a la condición de mero medio", op. cit., p. 136. 
PAULA ARIZPE

la apertura. Esta apertura consiste en un conocimiento por presencia en la profundidad (no en la superficie) que incluye, conjuntamente, la distancia de la reflexión y la inmediatez de la intuición. La apertura es la única categoría que puede albergar la aspiración humana del conocimiento creativo, que es tan amplia como la libertad. La verdad humana es la creencia en lo que todavía no somos pero podemos ser, una creencia en lo que no hemos hecho pero debemos hacer. La libertad es una aventura por realizar y que sólo puede conquistarse mediante una lucha cotidiana por existir. "Estoy en la búsqueda de la trascendencia una vez que esta profundidad se me ha abierto, y dentro de mi existencia temporal, el buscar se convierte en un encontrar: porque la existencia temporal del hombre como existencia-posible, sólo puede echar raíces en unidad con lo buscado, es decir, aquella presencia que existe sólo como búsqueda, que no se desvincula de lo buscado. Sólo mediante la intuición de lo buscado puede seguir adelante la búsqueda: la trascendencia está presente sólo cuando es buscada. Cuando se trasciende el ser objetual como sucede en la orientación intramundana (Weltorienturiung). No la aprehendo como me aprehendo a mí mismo, en Existenzerhellung, sino que tengo noción de ella por medio de una acción interior que, no obstante su fracaso, descansa en el auténtico ser. (...) Los modos de esta búsqueda del ser a nivel de una existencia posible son siempre caminos a la trascendencia. Su aclaración es metafísica", 5 ya que se trata del ámbito transobjetivo o reino de la libertad.

4 "En todos los tiempos el problema yace en la tensión: encontrar la independencia fuera del mundo, en renuncia y soledad o en el mundo mismo, a través del mundo, cooperando con él sin sucumbir a él. Así el filósofo sólo quiere su libertad junto con la libertad de los demás hombres, una vida sólo en comunicación con otros hombres", op. cit., p. 137.

${ }^{5}$ Philosophie, 1932, Berlín, Springer, vol. III, p. 3. Traducción libre. 
LA VERDAD EN JASPERS

\section{Pensamiento en suspensión o camino a la creatividad}

La metodología de Jaspers no constituye un camino lineal que va desde un punto de partida a otro sino, por el contrario, pretende enfatizar circular y tautológicamente los diferentes aspectos o perspectivas en los que cada fenómeno de la realidad se integra y bajo los cuales debe apreciarse en toda su magnificencia. Hemos de estar "abiertos" para permitir que la realidad nos impresione en toda su grandeza. Sólo por medio del pensamiento en suspensión ${ }^{6}$ cada objeto de conocimiento puede convertirse en un encuentro, que pueda incoar en nuestro ser más íntimo un acontecimiento creativo. Todo acontecimiento creativo engarza aspectos diversos de la realidad que el pensamiento científico y el filosófico tienden a disociar como 'contrastantes', 'dilemáticos', 'distintos' u 'opuestos'. Cuando un pensador adquiere el hábito de distinguir los diversos aspectos de la realidad y admirarlos en suspensión, en la multiplicidad de sus posibles relaciones sin apresurarse a escisiones insalvables, gana una sorprendente libertad para comprender genéticamente el fenómeno creativo; aquél en el que diferentes aspectos de la realidad y la temporalidad se vinculan mediante el poder creativo de la Existenz y lo comprometen con una instancia o ámbito apelante. Para calibrar todos los aspectos relevantes de la realidad es necesario tensar la mente para adaptarla al pensamiento en suspensión.

La vinculación a esta poderosa presencia de la trascendencia en la cual se sustenta toda la realidad relevante-que se impone de manera discreta como instancia suprema y absoluta-al hombre como infinitud e incondicionalidad o existente libre -búsqueda pensada y concomitantemente querida mediante la acción más íntima del sujeto-nos da la idea del movimiento circular de apelación-respuesta o ámbito nutricio de la realidad que caracteriza la metafisica jaspersiana. Esta profunda realidad genuinamente humana debe entenderse dentro de las categorías activo-receptivas mediales en las que se desarrolla la dialéctica del

${ }^{6}$ Denken in der Schwebe. 
juego. Es a esta realidad creativa, apelante, desafiante más palpable y vital para el existente a la que el autor invita.

El conocimiento profundo de la lógica de la creatividad permite al mismo tiempo aprehender los detalles de la lógica de la participación y del encuentro, y guiarnos directamente al descubrimiento de las realidades transobjetivas. ${ }^{7}$ Las realidades objetivas son aquellas mediante las cuales el existente se orienta dentro del mundo, objetos medibles, delimitables, verificables y cuantificables por todos mientras que las realidades transobjetivas son aquellas que tienen un estatuto espaciotemporal superior a la de los objetos empíricos y no pueden ser delimitadas o localizadas; sólo son captables para una existencia en pleno ejercicio de su libertad. Como no son situables a la distancia de un sujeto puramente cognoscente-puesto que comprometen a aquel que quiere aprehenderlas- le engloban, ya que ofrecen la posibilidad de profunda comunicación: de juego. No son objetivables porque no pueden ser proyectadas, sólo son aprehensibles por el modo de conocimiento participativo que vincula conocimiento y amor, conocimiento y acción creativa, conocimiento e inmersión activo-receptiva, en realidades relevantes que posibilitan la fundación de espacios lúdicos para penetrar (Ursprung) en el mundo transobjetivo, donde nada está 'dado', donde todo debe realizarse de una manera personal y creativa.

7 'Desde el principio hay en la filosofía algo inconmovible. En todo cambio en los estados y misiones de la existencia de la humanidad, en todo progreso de las ciencias, en todo desenvolvimiento de las categorías y métodos del pensamiento, se pretende aprehender la verdad eterna única en nuevas condiciones con medios nuevos, con posibilidades de claridad quizá mayores. Nuestra misión es actualmente cerciorarnos de nuevo de esta verdad eterna en el nihilismo llevado a su último extremo. Esto requiere que nos apropiemos de la tradición de un modo que no se limite a contemplar, sino que se sitúe en el interior como si fuera en la causa propia. (...) Lo que importa es apropiarse de la perfección alcanzada en cualquier época por la filosofía de suerte que permanezcamos en comunicación incesantemente renovada con los grandes fenómenos del pasado, no como superados sino como presentes", op. cit., p. 142-4. 
LA VERDAD EN JASPERS

\section{Ámbitos lúdicos de participación}

La creatividad es un fenómeno relacional iluminado por el ámbito de libre juego que se establece entre la Existenz y las realidades englobantes con las cuales funda una relación de encuentro comprometida. Entendido bajo este punto de vista creativo, el evento del juego significa la inmersión activo-receptiva en realidades que ofrecen multiplicidad de aspectos integrables, como forma y fondo, gracia y dignidad, movimiento y reposo. A diferencia de la postura kantiana, que tiende a disociar los diversos elementos y momentos de la experiencia, Jaspers pretende una profunda integración, en otros términos, superar la escisión sujeto-objeto a través de la creatividad práxica. Este movimiento integrador constituye un juego: una fecunda interrelación de elementos dotados de significado que, en el momento de conjuntarse se abren a la plenitud de sentido. De este modo la verdad es un alumbramiento que ocurre en un acontecimiento bajo una relación de encuentro, en la cual el objeto no solamente patentiza la forma que lo anima sino que distiende sus posibilidades interrelacionales. El ser humano no es sólo materia, como tampoco sólo espíritu. La verdadera humanidad sucede en el ámbito transobjetivo de la creatividad donde ambos elementos pueden conjuntarse de manera equilibrada, libre e inédita. La creatividad es el impulso para jugar. $Y$ el juego es aquello que no es contingente, ni en el sentido objetivo ni en el subjetivo, por lo que tampoco es coercitivo externa o internamente. Lo humano o existencial requiere para su cabal desarrollo y perfección abrirse a sí mismo a la realidad exterior y verdadera con todas sus normas y reglas (algo no contingente sino necesario). La aceptación de la normatividad deja de ser coactiva cuando la Existenz dotada con la voluntad de co-creación encuentra una gama de posibilidades de acción y las escoge y realiza, es decir, las hace suyas. Esta sólo aparente creatividad se torna grácil, luminosa y expresiva cuando ambas corrientes de la realidad -sujeto y objeto-se interrelacionan de manera lúdica formando un sólo ámbito: un juego expresivo. 
PAULA ARIZPE

\section{Integrar significa ser libre}

Como se ha visto, la Existenz se conforma en tanto espíritu a partir de la realidad objetiva, de las cosas materiales, unificándolas desde una perspectiva superior más libre y serena. Cuando la verdad empírica entronca con la verdad formal que da lugar a la ley de la necesidad, no se tensa por la abstracción disociante sino que se acompaña por la intuición inmediata de la creatividad.

Esta dialéctica de instalación en un horizonte o ámbito de comprensión de las realidades inmersas es paralelo a la dialéctica de la intuición y del discurso. Si por intuición se entiende la capacidad humana de penetrar de manera inmediata las realidades dotadas significación, y si el discurso se entiende como la sucesiva atención puesta a las diferentes perspectivas que estas realidades ofrecen, se puede observar una relación de mutua potenciación entre intuición y discurso, que nos lleva al reino de la intuición intelectual, siendo ésta tanto inmediata como indirecta (reflexiva). Esta original concepción puntualiza la mutua implicación de historicidad y verdad, temas medulares

90 en la reflexión jaspersiana, donde, los elementos mediales son asumidos por el existente con ímpetu creativo y se convierten en vehículos transparentes de presencia de la realidad que median. Esta concepción postula el carácter dinámico-relacional de la verdad y del proceso cognitivo, y por tanto funda el encuentro con la realidad como un reto creativo.

Nada es más íntimo al hombre que aquello que colabora a su plenitud, que contribuye a su propia realización como persona, y el impulso que lo incita a ello. En este sentido, los objetos son penetrados por la Existenz de manera directamente proporcional a como ésta se deja penetrar por los objetos de la realidad en toda su profundidad. Es una potenciación mutua basada en el esquema apelación-respuesta o circularidad del juego: sumergirse en una realidad englobante significa responder positivamente a una instancia valiosa y apelante. En otros términos, cuando existe comunidad de ideas el reino de la realidad se 
LA VERDAD EN JASPERS

torna familiar o habitable, es decir, pierde seriedad porque toma su tamaño adecuado; cuando se relaciona con la sensación y la necesidad pierde opacidad para convertirse en claro y simple.

Debido a su integratividad, el juego se ajusta y adapta constantemente, potencia la apertura mutua de posibilidades creativas. Crear calibrando las distintas posibilidades que la realidad abre a la Existenz es libertad auténtica. La seriedad cierra posibilidades; jugar implica "poner en juego" posibilidades de acción: ejercer la libertad.

La actividad lúdica supera el esquema sujeto-objeto, y en este hecho yace su profunda importancia ya que constituye un serio compromiso con la verdad. La auténtica seriedad al juego le viene de 'dentro', de la dialéctica juego-jugador, de la inmersión activa-receptiva del jugador en el ámbito de posibilidades que cada juego abre con su peculiar normatividad. El juego es serio porque es envolvente, el sujeto y el objeto son verdadera y comprometidamente uno dentro de un espacio y tiempo reales. Ésta constituye la verdad como totalidad aprehendida por la Existenz en el tiempo: El juego realiza en tanto es jugado; claramente no es un objeto, ya que lo que lo constituye no puede ser aprehendido por ningún intento especulativo. Un conocimiento tal sólo puede adquirirse en la profundidad de la experiencia lúdica. El jugador es un ámbito colmado de posibilidades de acción regido por normas lúdicas, y el juego es un ámbito de posibilidades de acciones con sentido que transforma al jugador en toda su integralidad. El carácter ambitalrelacional de la experiencia lúdica manifiesta su fecundidad en el orden de la praxis creativa. La realidad no está cerrada en sí misma sino que es precisamente un campo abierto para la creatividad interaccional. ${ }^{8}$ Éste es el significado que Jaspers intenta describir al usar el término "existencia posible": un ser que nunca es suficientemente pleno, que tiende a abrir su ámbito de realidad y que está llamado a ampliar su rango de acción en todas las direcciones. Por tanto, conceptualizado

8 "La disposición a una comunicación ilimitada no es consecuencia de un saber sino la resolución a tomar un camino en el ser-hombre. La idea de la comunicación no es utopía, sino fe", op. cit., p. 146. 


\section{PAULA ARIZPE}

de un modo integral, el ser humano es un ser ambital, abierto a desarrollarse mediante la constante fundación de relaciones comunicativas o ámbitos de juego.

\section{Habitar: unidad en la diversidad}

Así, la existencia se conforma mediante la creación de ámbitos. Esta empresa creativa presupone la permanencia que el 'habitar' implica. Todo lo valioso que el hombre realiza se debe a la permanencia, $y$ para permanecer es necesario echar raíces en buena tierra, es decir: habitar. La creación humana de ámbitos -ámbitos de conocimiento, amistad, amor, arte, etc-- corre paralela a la creación de espacios físicos que son de cierto modo expresión del dinamismo humano del habitar. Estos espacios son genuinamente habitables cuando pueden recrearse por personas que los habitan cotidianamente. ¿Cómo es esto posible? Hay espacios dentro de los cuales el hombre se encuentra 'en casa', y en los cuales siente una sutil adecuación -absolutamente perceptible y dificilmente analizable-que se da entre sus condiciones espirituales y

92 el entorno. Ciertas viejas ciudades, plazas, atmósferas domésticas o sitios sagrados suscitan en el hombre un agradable sentimiento de equilibrio y armonía. Esta coordinación armónica se produce cuando el hombre recrea en su cotidianidad los espacios que estructuran la trama de su existencia diaria, con lo que convierte un espacio físico en verdadero ámbito humano. El espacio real, el único auténticamente humano es el configurado por una comunidad a través de su vivir cotidiano. No hay nada extraño en sentir un confortable estado de paz - fruto de la plenitud- al visitar algunas viejas ciudades creadas lentamente por sus habitantes, desarrolladas orgánicamente desde dentro hacia fuera, en conformidad con sus requerimientos intrínsecos o lógica propia. En la vida de una comunidad el espacio representa un papel análogo al del tiempo en una sinfonía: ambos constituyen un ámbito natural y necesario de desdoblamiento, un horizonte en el cual el hombre puede expandir sus posibilidades. 
Estos ámbitos no son 'dados' a la Existenz por ley natural; ésta tiene que fundarlos libremente en la tensa distensión que la vida en comunidad implica, ya que el espacio vital no es estático -como se piensa comúnmente- sino que se conforma por un entramado muy complejo de interrelaciones dinámicas y tensiones internas. La posible existencia debe decidir y así realizarse a sí misma para devenir Existenz: éste es el auténtico reino de la libertad. "El Ser-sí-mismo (Selbst) se relaciona con sí-mismo (Selbst) de una manera creativa": ${ }^{9}$ éste es el reto humano. Este marco de interrelaciones constituye un orden, una ordenación, un sentido. El orden ha de ser aprehendido y revivido por el hombre que habita un espacio, si éste ha de ser rigurosamente 'habitable' debe producir tanto equilibrio como un sentimiento de paz sin provocar una relajación disolvente sino al contrario, una profunda integración del espíritu. Consecuentemente, cualquier estudio sobre el espacio se convierte en una alabanza al orden, entendido positivamente como una ordenación eminentemente activa. En este punto se conforma el círculo virtuoso: el dinamismo de la existencia humana confiere una ordenación y un sentido al espacio, y el espacio vitalmente estructurado hace posible una vida con sentido. La existencia es tal cuando se mueve dentro de un determinado espacio en el entramado de las interrelaciones humanas. Para el autor ésta es la razón por la cual el hombre se pierde inevitablemente en el magma amorfo de un mundo sin límites (existencia empírica), falto del sentido que sólo la inmersión creativa o la delimitación existencial confiere.

La existencia no puede vivir estática o pasivamente en un espacio: la Existenz tiene una necesidad originaria de insertarse activamente en un ámbito - envolvente-configurándose y comunicándose, con todo el dramatismo que su existencia implica.

Jaspers pretende delimitar la realidad ambital de la existencia, ya que éste es el único situs adecuado donde puede realizar las propias posibilidades: ejerciendo su libertad al encuentro con la existencia. Este encuentro con el propio ser es un contacto creador a nivel esen-

${ }^{9}$ Philosophie, op. cit., vol. II, p. 32. 
PAULA ARIZPE

cial. El hombre se encuentra inclinado originariamente a la fundación de nuevos ámbitos en colaboración con otros seres. Estos ámbitos son tan fuertes y valiosos como profundos sean los seres que los conforman relacionalmente; cuanto más cerca se hallen éstos de su propio origen, mientras más sí mismos sean. Así es como el ámbito de la libertad es ensanchado por la existencia. Por lo tanto, la creación de ámbitos dialógicos es la actividad primaria y más alta de la existencia: el ser humano deviniendo tal. El hombre es positivamente espacial porque tiene la capacidad de crear ámbitos de interrelación, para los cuales la distensión física representa sólo la plataforma material y obvia desde la cual construir o conquistar la existencia. Los ámbitos son el resultado de la acción configurativa del mundo, entendiendo a éste como un campo abierto de posibilidades no como un mero espacio físico.

Jaspers dejó claro que la empírica es sólo el presupuesto de la existencia posible. Y la posible existencia deviene Existenz en la lucha cotidiana de dirigirse, ordenando su libertad, hacia la trascendencia, es decir, creando ámbitos donde se realice a sí misma ampliamente. Por ello, la dinamicidad de la humana existencia está orientada hacia la creación de ámbitos. Para el autor, existir es ejercer la libertad, y la libertad en sentido absoluto es relacionalidad o comunicación.

\section{La verdad como praxis creativa}

Jaspers en La fe filosófica y Filosofia describe al salto (Ursprung) a la trascendencia como el núcleo de la realización de la existencia posible del hombre. Este salto que significa la suprema y radical inmediatez con lo real es el modo más eminente de existencia, y puesto que no está 'dado', como lo está el Dasein, debe hacerse de manera co-creativa a través de todos los modos de comunicación con los objetos de la realidad, es decir con lo envolvente (Das Umgreifende).

El autor muestra que las formas más elevadas de la realidad envolvente son aquéllas que requieren de la Existenz la comunicación por medio de los modos más vigorosos de creatividad para, en conse- 
cuencia, fundar los géneros más perfectos de inmediatez. Estos modos de inmediatez son sólo posibles en el corazón de una cercanía presencial y envolvente a través de una búsqueda confiada. Por otro lado, si el hombre en búsqueda de seguridad se acoge a métodos objetivos, adecuados tan sólo a realidades objetivas (mesurables, no envolventes, meramente problemáticas porque no comprometen) hace que la trascendencia desaparezca. ${ }^{10}$

Por ende, la verdad es un evento ineludible. La verdad es inmersiva, co-creativa, lúdica, es un impulso a la acción/realización, nunca estática: La verdad es lo envolvente.

La comprensión de la verdad no es una mera técnica de interpretación, sino una praxis dialógica e interaccional en la cual la existencia se compromete libremente con la realidad y sus ámbitos. El hombre está abierto más allá de lo dado. Por ello, entre nuestra actividad comprehensiva y nuestra apertura a la totalidad hay una relación circular y creativa: Sólo tenemos que aventurarnos a existir creativamente.

${ }^{10}$ Cfr., Philosophie, op. cit., vol. III, p. 8. 